\title{
Reconstrução de membros inferiores após excisão de úlcera de Marjolin: relato de casos
}

\section{Lower extremities reconstructions after Marjolin's ulcer excision: cases report}

\author{
Katia Torres Batista ${ }^{1}$ \\ Hugo José de Araújo \\ Aloysio CAMPOS DA \\ PAZ JUNIOR ${ }^{3}$
}

Trabalho realizado na Rede Sarah de Hospitais de Reabilitação, Brasília, DF, Brasil.

Artigo submetido pelo SGP (Sistema de Gestão de Publicações) da RBCP.

Artigo recebido: $28 / 5 / 2009$ Artigo aceito: 5/8/2009

\begin{abstract}
RESUMO
Introdução: A úlcera de Marjolin é uma rara complicação de cicatrizes crônicas, mais comum após queimaduras, havendo predominância nos membros inferiores. Objetivo: Descrever uma série de pacientes com úlcera de Marjolin nos membros inferiores, atendidos no Hospital Sarah Brasília entre 2001-2008. Relato dos casos: Foram realizadas a excisão tumoral e a reconstrução com enxertia de pele; retalho sural reverso, amputação e programa de reabilitação. A amputação foi considerada nos casos em que não foi possível a reconstrução. Foi diagnosticado carcinoma epidermóide bem diferenciado por biopsia de congelação transoperatória e exame histopatológico. Não houve relato de recidiva após cinco anos. Conclusão: Os autores recomendam o seguimento e a avaliação de cicatrizes instáveis com biopsia em intervalos regulares. Nos casos diagnosticados de câncer realizar a excisão completa e atenção para as adaptações funcionais com órteses e próteses.
\end{abstract}

Descritores: Úlcera. Úlcera cutânea. Queimaduras/complicações.

\section{SUMMARY}

Introduction: Marjolin's ulcer is a rare complication of chronic scars that is most often associated with burn wounds. There was predominance developing in the lower extremities. Objective: To describe results in a serie of cases with Marjolin's ulcer in lower extremity admitted in the Hospital Sarah Brasilia, between 2001-2008. Case reports: We performed wide tumoral excision and reconstruction with grafts, sural reverse flap, amputation and rehabilitation program. Amputation was considered in cases where surgical excision is not possible. Differentiated squamous cell carcinoma was diagnosis by frozen section biopsy and histopathological analyze. There were no reports of disease recurrence after five years. Conclusion: The authors recommend the follow up and that unstable scars were evaluated with biopsy at regular intervals. If cancer is found wide excision is indicated and attention for functional adaptations with orthesis or prosthesis.

Descriptors: Ulcer. Skin ulcer/etiology. Burns/complications.

1. Membro titular da Sociedade Brasileira de Cirurgia Plástica (SBCP); Médica Cirurgiã Plástica do Hospital Sarah Brasília.

2. Membro titular da SBCP, Médico Cirurgião Plástico do Hospital Sarah Brasília.

3. Doutor em Ortopedia; Cirurgião-chefe da Rede Sarah de Hospitais de Reabilitação. 


\section{INTRODUÇÃO}

Úlcera de Marjolin é o termo usado para descrever a degeneração maligna para carcinoma epidermóide, que ocorre em áreas de processo inflamatório crônico de cicatrizes instáveis pós-queimaduras. É descrita na literatura como um tumor de comportamento agressivo, alta recorrência, com possibilidade de metástases para linfonodos. $\mathrm{O}$ tempo entre a queimadura e o surgimento do tumor é, em média, de 30 anos.

Quando uma úlcera no membro inferior não responde ao tratamento clínico, ou cicatriza por segunda intenção, com instabilidade e ferimentos repetidos, o potencial para degeneração maligna existe. É importante considerar o diagnóstico e propor apropriada intervenção terapêutica, com ressecção completa com margens cirúrgicas livres e rastreamento para metástases, principalmente para linfonodos.

Não existe consenso quanto à efetividade da cirurgia na cura do tumor, entretanto, na maior parte da literatura pertinente, é ressaltada a importância da ressecção cirúrgica ampla e radical, como apontado na revisão sistemática de 443 casos, publicada por Valentik et al. ${ }^{1}$.

O objetivo desse trabalho é descrever a reconstrução e evolução de uma série de pacientes, após excisão da úlcera de Marjolin no membro inferior.

\section{RELATO DOS CASOS}

Dentre os 120 pacientes operados pela equipe de Cirurgia Plástica do Hospital Sarah/ Brasília, entre janeiro de 2001 e dezembro de 2006, devido a sequela de queimadura, quatro $(2,7 \%)$ tiveram o diagnóstico de úlcera de Marjolin nos membros inferiores (Tabela 1).

Os procedimentos cirúrgicos foram realizados sob anestesia locorregional ou geral, drenagem venosa por elevação do membro e uso de garrote pneumático. A excisão da tumoração foi feita com margens macroscópicas de $2 \mathrm{~cm}$, livres de tumor, após estudo por congelação transoperatório e confirmado pelo exame histopatológico pós-operatório, identificando-se as margens cirúrgicas livres.

O critério para indicação da reconstrução variou conforme o defeito e a localização. Realizou-se a regularização de amputação transtibial na lesão localizada no coto de amputação da perna; amputação transtibial na lesão localizada no pé, enxertia de pele naquela localizada no cavo plantar e retalho sural reverso em tumor localizado no calcâneo.

Na investigação oncológica, realizou-se exames de radiografia e tomografia computadorizada do tórax, todos com resultados normais.

Foram aplicadas próteses provisórias no pós-operatório imediato, visando ao exercício sensório e à prevenção da "dor fantasma". Para auxílio à locomoção foram confeccionadas palmilhas sob molde para alívio de pressão no cavo plantar; órtese com apoio no tendão patelar (PTB) e prótese transtibial para membros inferiores.

O seguimento pós-operatório foi realizado por meio de revisões trimestrais, semestrais e anuais, com avaliação local, linfonodos, radiografia e tomografia computadorizada de tórax.

As Figuras 1 a 5 ilustram os casos apresentados nesse estudo.

\section{DISCUSSÃO}

Observou-se que o tempo de latência, semelhantemente ao que foi descrito pelos autores pesquisados, é longo e varia de 25 a 40 anos, média de 29 anos s.3-5 $^{1,3}$. Embora tenha sido descrita uma taxa de recorrência de $14,7 \%^{1}$, esta não foi observada nos casos que compõem a presente casuística, com período de seguimento pós-operatório entre 3 e 9 anos. Está descrita a alta agressividade desses tumores ${ }^{1,7,8}$, principalmente no local, assim, há

Tabela 1 - Caracterização dos pacientes.

\begin{tabular}{|c|c|c|c|c|c|c|c|}
\hline Paciente & Idade & Sexo & Mecanismo & $\begin{array}{c}\text { Tempo entre a } \\
\text { queimadura e o } \\
\text { tumor (latência) }\end{array}$ & Localização & Tratamento & $\begin{array}{l}\text { Tempo de } \\
\text { seguimento e } \\
\text { programa de } \\
\text { reabilitação }\end{array}$ \\
\hline 1 & 27 & M & fogo & 26 anos & calcâneo & $\begin{array}{c}\text { E }(8 \times 7 \times 2 \mathrm{~cm})+\text { biopsia } \\
\text { de linfonodos } * \\
+ \text { RSR }\end{array}$ & $\begin{array}{c}4 \text { anos } \\
\text { órtese PTB }\end{array}$ \\
\hline 3 & 43 & $\mathrm{~F}$ & fogo & 42 anos & $\begin{array}{l}\text { coto de } \\
\text { amputação } \\
\text { plantar }\end{array}$ & $\begin{array}{c}\text { Amputação } \\
\text { transtibial }\end{array}$ & $\begin{array}{l}3 \text { anos } \\
\text { prótese transtibial } \\
\text { imediata }\end{array}$ \\
\hline
\end{tabular}

*biopsia de linfonodos inguinais com resultado histopatológico negativo; E: Excisão; RSR: Retalho sural reverso. 


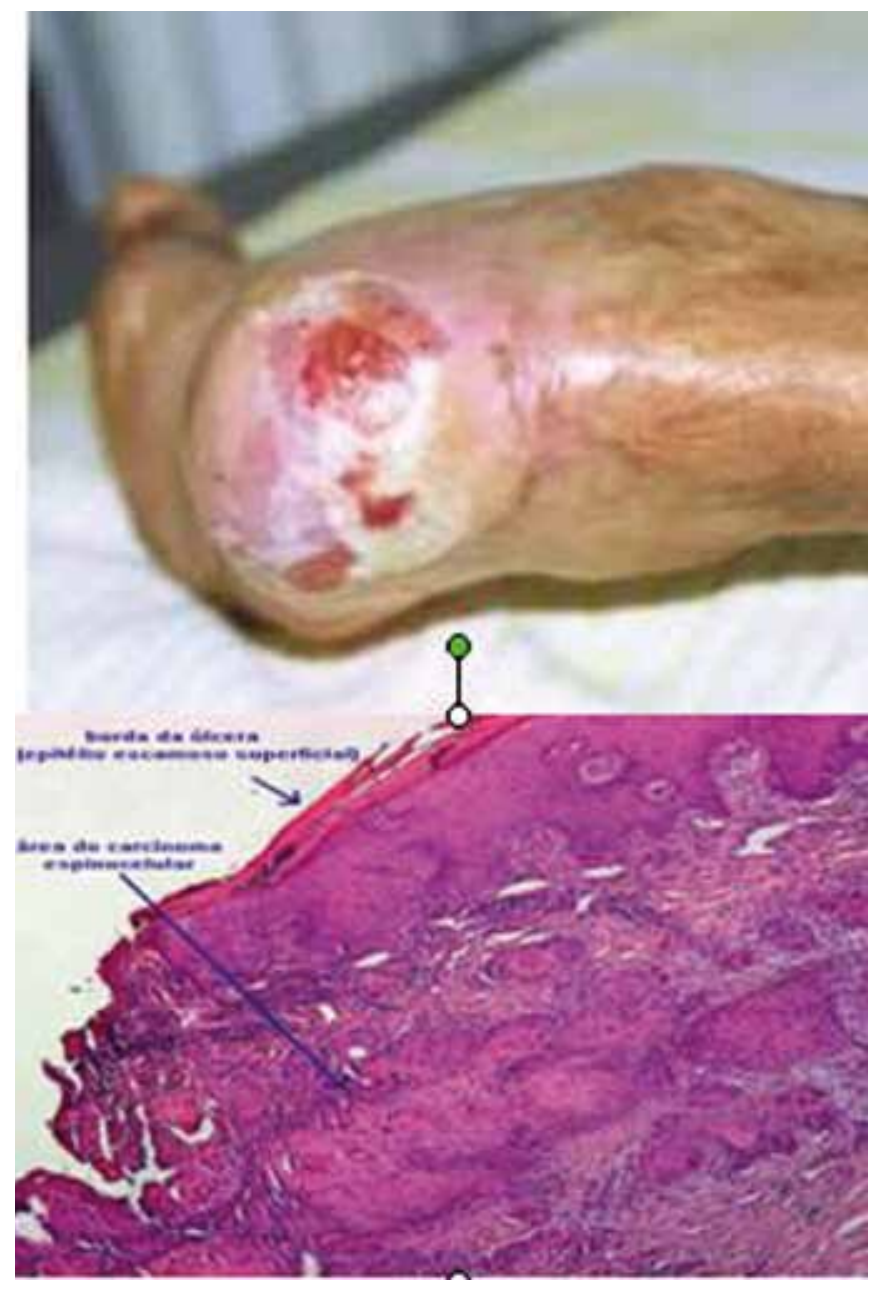

Figura 1 - Caso clínico 1. Paciente apresentando úlcera na região calcânea, aspecto pré-operatório (A) e histopatológico (B).

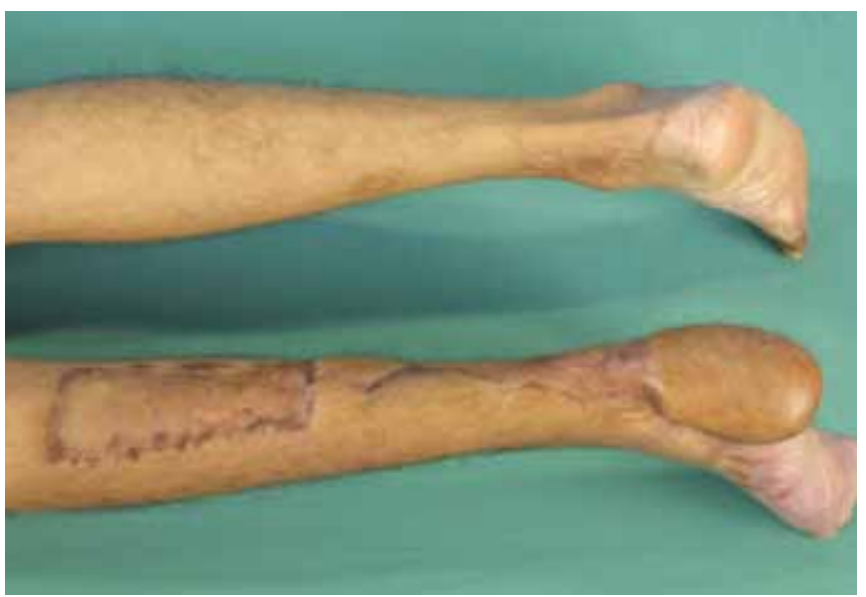

Figura 2 - Caso clínico 1. Aspecto pós-operatório após um ano do procedimento de ressecção e reconstrução com retalho sural reverso em ilha.

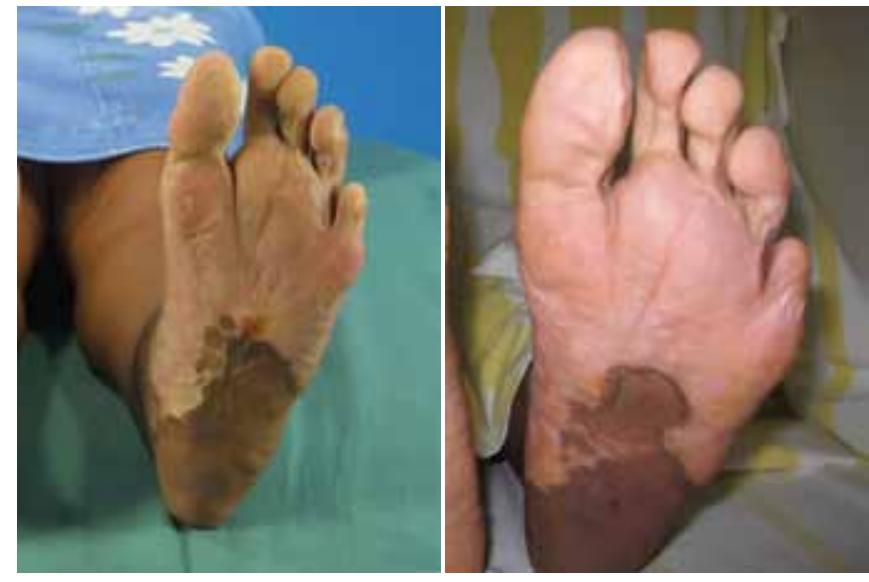

Figura 3 - Caso clínico 2. Paciente apresentando úlcera no cavo plantar no pré-operatório e no período pós-operatório, após excisão e enxertia de pele total.

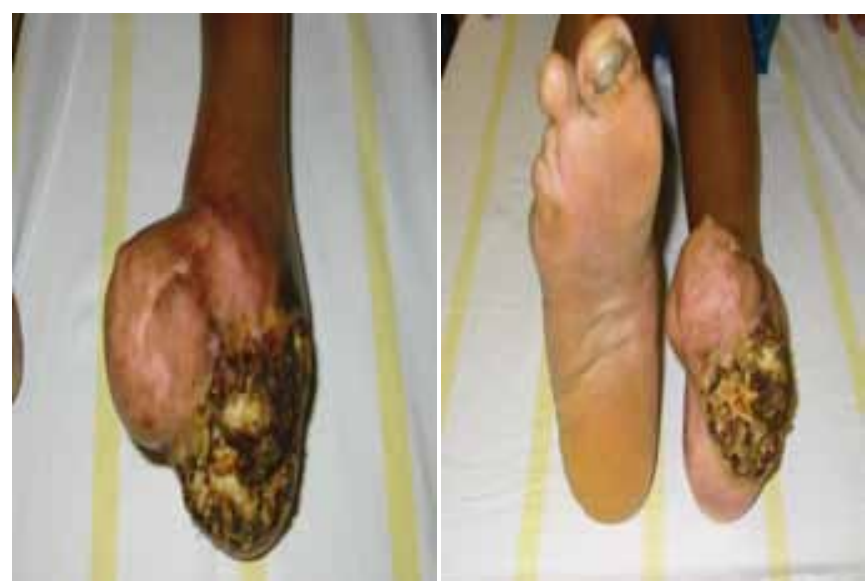

Figura 4 - Caso clínico 3. Degeneração maligna no coto de amputação do pé esquerdo; realizada amputação transtibial e protetização imediata.
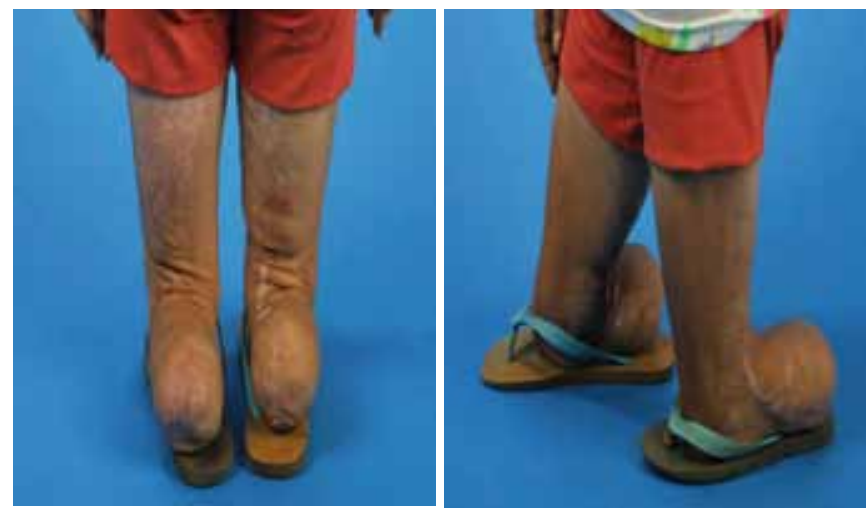

Figura 5 - Caso clínico 4. Paciente apresentando amputação das pernas por queimadura, com adaptação funcional pela própria paciente. Evoluiu com úlcera no coto de amputação direito. Foi realizada excisão e regularização de coto de amputação transtibial. 
necessidade de realização de diagnóstico precoce e adoção de medidas preventivas, como a melhor cobertura, inspeção e proteção das cicatrizes de queimaduras nos membros inferiores. Sempre que houver suspeita da degeneração maligna, deve-se realizar uma biopsia da região, e se esta for positiva, proceder à excisão ampla e à reconstrução com pele de boa qualidade, sobretudo nos membros inferiores, situação em que há necessidade de reabilitação, com o uso de órteses e próteses.

$\mathrm{Na}$ fase pós-operatória, o programa de reabilitação enfoca o posicionamento, os curativos compressivos, o controle do edema e o fortalecimento muscular com o objetivo de preparálos para o encaixe da prótese. A confecção e a seleção dos materiais protéticos foram realizadas, considerando as necessidades individuais, dependendo do nível ou do tipo de perda do membro. No início da fase protética, nos casos apresentados, a partir da quarta semana de pós-operatório, realizou-se o ajuste do coto e dos anexos protéticos, o alinhamento biomecânico e estático das próteses, a avaliação dinâmica, verificando se havia irritação na pele ou dificuldades durante o uso das próteses. Após a protetização imediata, na fase de continuidade, realizou-se realinhamento e procedimentos de modificação, os pacientes receberam treinamento protético, visando ao equilíbrio e atividades de deambulação (andar, subir e descer escadas e declives, atravessando obstáculos, pegar objetos do chão, subir e descer de um veículo).

Os ajustes e revisões são fundamentais na prevenção de úlceras nos cotos amputados, as quais normalmente cicatrizam, em média, em seis semanas após a suspensão do uso da prótese. Vale ressaltar a existência de problemas relacionados aos cotos de amputação, tais como a cicatrização, o "membro fantasma", as desordens cutâneas e aquelas relacionadas à imagem corporal.

As revisões regulares são fundamentais, principalmente nos pacientes com história de amputação por doença vascular e degeneração de úlceras crônicas. Estudos do Prosthetic and Amputee Rehabilitation Centre at Chapel Allerton Hospital $(\mathrm{CAH})^{9}$ revelaram que $43 \%$ dos pacientes em uso de próteses têm problemas com o coto de amputação, dentre eles as úlceras com prevalência de $20 \%$. Nos casos amputados, aqui apresentados, realizou-se a protetização imediata, com os pacientes ainda anestesiados e a instalação de bomba de analgesia peridural contínua para a prevenção da "dor fantasma". No estudo realizado por Campos da Paz e Braga, em pacientes amputados por sarcoma, pode-se afirmar que o fenômeno do membro fantasma não é um sintoma relacionado ao coto de amputação, pois se verificou que o cérebro não foi danificado e as suas áreas de comando e percepção ainda estavam intactas e enviando estímulos como se o membro ainda estivesse presente. Considerouse que a montagem de uma prótese exige não só a tecnologia, mas também uma abordagem diferenciada e humanística, onde os pacientes com bons ajustes apresentaram melhores resultados nos estudos no laboratório de movimento e na avaliação neuropsicológica.
Por fim, a sensação de ter "perdido" parte do corpo pode ser usada para a montagem de uma prótese imediatamente após a amputação, enquanto o paciente ainda está sob anestesia, e, em seguida, de forma contínua, iniciar a formação do estímulo vibratório, como tem sido feito pela Rede SARAH de Hospitais, pode se permitir a preservação da percepção sensorial no córtex e se tornar um fator importante para evitar a dor. O membro inferior, embora artificial, ainda é percebido como se estivesse lá ${ }^{10}$.

Segundo Dutra et al. ${ }^{8}$, o principal fator para a sobrevida de portadores de úlcera de Marjolin é o grau de diferenciação visto no exame histopatológico. Consideramos importante o estudo de congelação transoperatório para obtenção de margens cirúrgicas livres e avaliação do grau de diferenciação para definição do acompanhamento oncológico.

\section{CONCLUSÃo}

Os autores descrevem uma série de casos de indivíduos portadores de úlcera de Marjolin nos membros inferiores, cujo exame histopatológico revelou pequeno grau de diferenciação. Sugerem a realização de biopsia por congelação transoperatória, excisão com margens cirúrgicas livres de tumores, reconstrução e programa de reabilitação precoce.

\section{REFERÊNCIAS}

1. Kerr-Valentic MA, Samimi K, Rohlen BH, Agarwal JP, Rockwell WB. Marjolin's ulcer: modern analysis of an ancient problem. Plast Reconstr Surg. 2009;123(1):184-91.

2. Smith J, Mello LF, Nogueira Neto NC, Meohas W, Pinto LW, Campos VA, et al. Malignancy in chronic ulcers and scars of the leg (Marjolin's ulcer): a study of 21 patients. Skeletal Radiol. 2001;30(6):331-7.

3. Bauk VOZ, Assunção AM, Domingues RF, Fernandes NC, Maya TC, Maceira JP. Úlcera de Marjolin: relato de 12 casos. An Bras Dermatol. 2006;81(4):355-8.

4. Asuquo M, Ugare G, Ebughe G, Jibril P. Marjolin's ulcer: the importance of surgical management of chronic cutaneous ulcers. Int $\mathrm{J}$ Dermatol. 2007;46(Suppl 2):29-32.

5. Tank J, Scharschmidt T, Weiner SD. Development of bilateral lower extremity Marjolin ulcers after childhood burns. Am J Orthop (Belle Mead NJ). 2008;37(6):E113-5

6. Akhtar S, Hameed A. Versatility of the sural fasciocutaneous flap in the coverage of lower third leg and hind foot defects. J Plast Reconstr Aesthet Surg. 2006;59(8):839-45.

7. Kakagia D, Tamiolakis D, Grekou A, Vavetsis S, Lambropoulou M, Papadopoulos N. Intraoperative cytological evaluation of Marjolin ulcers. Onkologie. 2006;29(1-2):21-4.

8. Dutra AK, Gomes FQ, Meza GLF, Rodrigues RC, Possari E, Rstom SA, et al. Úlcera de Marjolin em tórax: relato de caso. Rev Soc Bras Cir Plast. 2008;23(2):131-4.

9. Wadwhani S. Prevalence of stump problems in a community sample of lower limb amputees. Leeds: Leeds Medical School SSM Project;2001.

10. Campos da Paz Jr A, Braga LW, Downs JH 3rd. A preliminary functional brain study on amputees. Appl Neuropsychol. 2000;7(3):121-5. 